\title{
A Mathematica Package for Solving and Displaying Inequalities
}

\author{
R. Ipanaqué ${ }^{1}$ and A. Iglesias ${ }^{2 \star}$ \\ 1 Mathematics Department, National University of Piura, Perú \\ robertchero@hotmail.com \\ 2 Department of Applied Mathematics and Computational Sciences, University of \\ Cantabria, Avda. de los Castros, s/n, E-39005, Santander, Spain \\ iglesias@unican.es \\ http://personales.unican.es/iglesias
}

\begin{abstract}
Solving inequalities is a very important topic in computational algebra. In fact, the most important computer algebra systems include sophisticated tools for solving different kinds of inequalities in both symbolic and graphical ways. This paper presents a new Mathematica package, InequationPlot, for displaying the two-dimensional solution sets of several inequalities. In particular, it extends Mathematica's capabilities by providing graphical solutions to many inequalities (such as those involving trigonometric, exponential and logarithmic functions) that cannot be solved by using the standard Mathematica commands and packages. The package also deals with inequalities involving complex variables by displaying the corresponding solutions on the complex plane. The output obtained is consistent with Mathematica's notation and results and the package provides a similar output for those cases already solved by Mathematica. The performance of the package is discussed by means of several illustrative and interesting examples.
\end{abstract}

\section{Introduction}

Solving inequalities is a very important topic in Mathematics, with outstanding applications in many problems of theoretical and applied science. Inequalities play a key role simply because many problems cannot be completely and accurately described by only using equalities. However, since there is not a general methodolody for solving inequalities, their symbolic computation is still a challenging problem in computational algebra. Depending on the kind of the functions involved, there are many "specialized" methods such as those based on cylindrical algebraic decomposition, Gröebner basis, quantifier elimination, etc. In addition, some "ad hoc" methods are to be implemented. The interested reader is referred to [13/457] for an introduction to the field. See also [8] for a recent survey on geometric inequalities.

In spite of the difficulties mentioned above, the most important computer algebra systems (Mathematica, Maple, etc.) include sophisticated tools for solving

\footnotetext{
* Corresponding author
} 
different kinds of inequalities in both symbolic and graphical ways. However, the problem is far from being solved in all its generality and, consequently, there is still a long way to walk in order to tackle this issue.

This paper presents a new Mathematica package, InequationPlot, for displaying the two-dimensional solution sets of of several inequalities. In particular, it extends Mathematica's capabilities by providing graphical solutions to many inequalities (such as those involving trigonometric, exponential and logarithmic functions) that cannot be solved by using the standard Mathematica commands and packages. The package also deals with inequalities involving complex variables by displaying the corresponding solutions on the complex plane. The output obtained is consistent with Mathematica's notation and results and the package provides a similar output for those cases already solved by Mathematica. The performance of the package is discussed throughout the paper by means of several illustrative and interesting examples.

The structure of this paper is as follows: Section 2 describes the main standard Mathematica tools for solving inequalities. Then, Section 3 introduces the new Mathematica package, InequationPlot, and describes the commands implemented within. The performance of the package is also discussed in this section by using some illustrative examples. In addition, we show some inequalities that are solvable with our package while they cannot be solved by applying the standard Mathematica kernel and packages. Finally, Section 4 closes with the main conclusions of this paper and some further remarks.

\section{Standard Mathematica Tools for Solving Inequalities}

Mathematica incorporates a number of sophisticated commands and packages for solving different kinds of inequalities. For example, the Mathematica command Reduce [eqns,vars, elims] simplifies equations trying to eliminate the variables elims. In this process, the command generates both equations and inequalities combined with the operators \&\& (and) and II (or). In addition, Mathematica 3.0 includes a package, Algebra'AlgebraicInequalities', for solving systems of strong polynomial inequalities [9]. In particular, the command SemialgebraicComponents $\left[\left\{e_{1}, \ldots, e_{n}\right\},\left\{x_{1}, \ldots, x_{m}\right\}\right]$ gives at least one point in each connected component of the open semialgebraic set defined by inequalities $\left\{e_{1}, \ldots, e_{n}\right\}$, where both sides of each $e_{i}$ are polynomials in variables $\left\{x_{1}, \ldots, x_{m}\right\}$ with rational coefficients. This command is based on the cylindrical algebraic decomposition algorithm (see, for instance, 2] and [6] for a description). In addition, the Mathematica 3.0 package Algebra'InequalitySolve' provides the solution set of an expression containing logical connectives and univariate polynomial equations and inequalities in a single or several variables. Other recent commands to deal with this problem incorporated in Mathematica version 4.0 are described in [10. That paper also discusses the main algorithms used in this task.

The visualization of the solution sets of given systems of inequalities has also been the subject of further research. For example, the add-on Mathematica 
4.1 package Graphics 'InequalityGraphics' provides commands to visualize logical combinations of polynomial and algebraic inequalities in two- and threedimensional real spaces (commands InequalityPlot and InequalityPlot3D respectively). Some additional developments to find real solutions of systems of real equations and inequalities have also been recently incorporated into Mathematica version 5.0. For example, the command FindInstance can be used to find a real solution of a system of equations and inequalities. Even users can directly apply the CylindricalDecomposition command to compute solutions of certain kinds of inequalities.

However, there are several families of inequalities that cannot be solved by using the standard Mathematica commands described above. This limitation can easily be understood by analyzing the methods applied to solve those inequalities, mostly based on the cylindrical algebraic decomposition (CAD). In this decomposition, each $S \subset \mathbb{R}^{n}$ is represented as a finite union of disjoint cells. The concept of cell applied here can be defined as follows:

1. a cell in $\mathbb{R}$ is either a point or an open interval

2. a cell in $\mathbb{R}^{n+1}$ is either defined by the set

$$
\{(x, y) / x \in C, f(x)<y<g(x)\}
$$

or the set

$$
\{(x, y) / x \in C, y=f(x)\}
$$

where $x=\left(x_{1}, x_{2}, \ldots, x_{n}\right), C$ is a cell in $\mathbb{R}^{n}$ and $f$ and $g$ are either

a) continuous functions on $C$ such that for some polynomials $F$ and $G$ we have $F(x, f(x))=0$ and $G(x, g(x))=0$ or

b) $\pm \infty$

and $f(x)<g(x), \forall x \in C$.

Given a finite set $F$ of polynomials in $n$ variables, the CAD algorithm calculates an $F$-invariant 11 cylindrical algebraic decomposition of $\mathbb{R}^{n}$. This algorithm is particularly well suited for obtaining the solution set of a system of polynomial equations and inequalities for $n$ variables. For example:

$\operatorname{In}[1]:=$ CylindricalDecomposition $\left[\mathrm{x}^{\wedge} 2+\mathrm{y}^{\wedge} 2+\mathrm{z}^{\wedge} 2<1,\{\mathrm{x}, \mathrm{y}, \mathrm{z}\}\right]$

$$
\begin{aligned}
\text { Out }[1]:= & \left\{-1<x<1 \& \&-\sqrt{1-x^{2}}<y<\sqrt{1-x^{2}} \& \&\right. \\
& \left.-\sqrt{1-x^{2}-y^{2}}<z<\sqrt{1-x^{2}-y^{2}}\right\}
\end{aligned}
$$

Unfortunately, as will be shown later, inequalities involving trigonometric, exponential or logarithmic functions cannot be decomposed in cells and, therefore, the CAD method can no longer be applied to those cases. The package InequationPlot, described in the next section, overcomes this limitation and allows the user to solve a large family of real and complex inequality systems and equations and display their two-dimensional solution sets.

\footnotetext{
${ }^{1}$ A cylindrical algebraic decomposition of a set $S \subset \mathbb{R}^{n}$ is said to be $F$-invariant if each of the polynomials from $F$ has a constant sign on each cell of that decomposition.
} 


\section{The Package InequationPlot: Some Illustrative Examples}

Inequalities involving trigonometric functions cannot be solved by applying the CAD algorithm described in Section 2. For example, let us try to display the solution sets of each of the inequalities

$$
\sin (x+y)>\frac{1}{2}
$$

and

$$
\sin (2 x)+\cos (3 y)<1
$$

on the set $[-8,8] \times[-8,8]$ by using the standard Mathematica commands. In this case, we must use the command InequalityPlot of the Mathematica 4.1 package:

In [2] : =<<Graphics 'InequalityGraphics '

Unfortunately, since the region defined by inequality (3) on the prescribed domain cannot be broken down into cylinders, the CAD algorithm fails to give the solution:

$\operatorname{In}[3]:=\operatorname{InequalityP} \operatorname{lot}[\operatorname{Sin}[\mathrm{x}+\mathrm{y}]>1 / 2,\{\mathrm{x},-8,8\},\{\mathrm{y},-8,8\}]$

Out $[3]:=$ InequalityPlot :: region :

The region defined by $\sin (x+y)>1 / 2 \bigwedge-8<=x<=8 \wedge-8<=y<=8$ could not be broken down into cylinders.

The previous inequalities can be solved, however, by loading the package developed by the authors:

In $[4]:=<<$ InequationPlot

which includes the command

InequationPlot [ineqs, $\{x, x \min , x \max \},\{y, y \min , y \max \}$, opts]

for displaying the two-dimensional region of the set of points satisfying the inequalities ineqs of real numbers inside the square [xmin, xmax] $\times$ [ymin, ymax $]$, which is actually the problem we are going to solve. For example, inequalities (3)-(4) can be solved as follows:

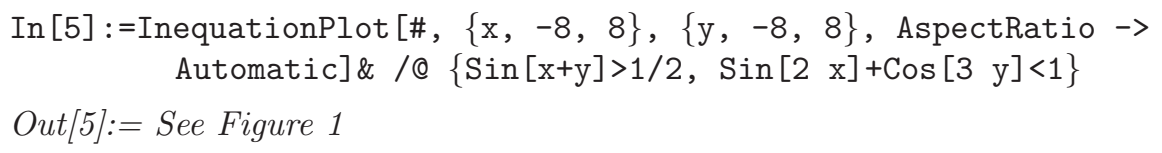

Similarly, Fig. 2 displays the solution sets of the inequalities $F(x)+F(y)=1$ and $F\left(x^{2}\right)+F\left(y^{2}\right)=1$ (where $F$ stands for the floor function) on the squares $[-4,4] \times[-4,4]$ and $[-2,2] \times[-2,2]$, respectively. We would like to remark that the Mathematica command InequalityPlot does not provide any solution for these inequalities either. 

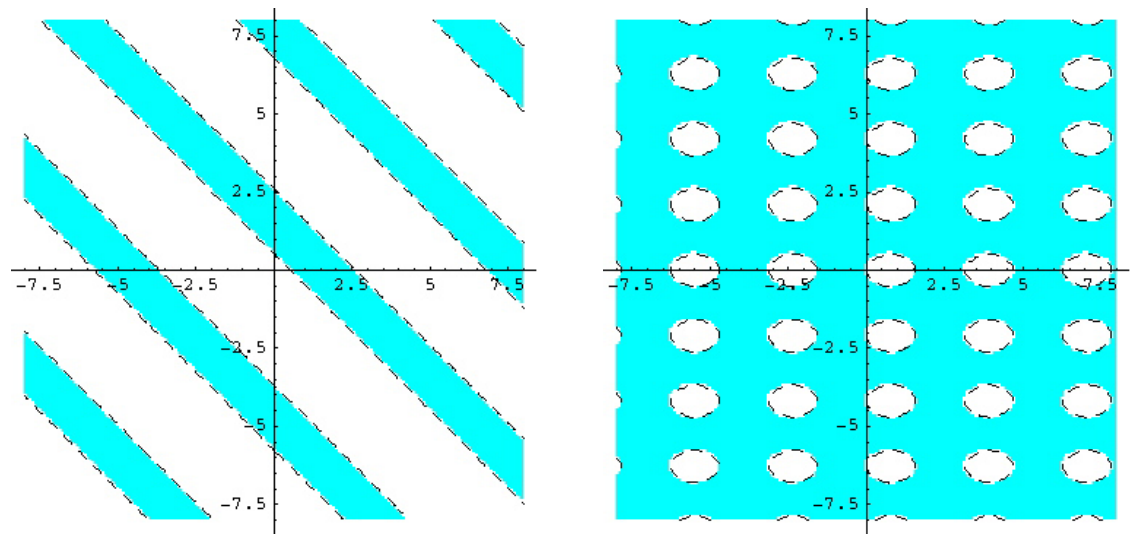

Fig. 1. Some examples of inequality solutions on the square $[-8,8] \times[-8,8]$ : (left) $\sin (x+y)>\frac{1}{2} ;$ (right) $\sin (2 x)+\cos (3 y)<1$
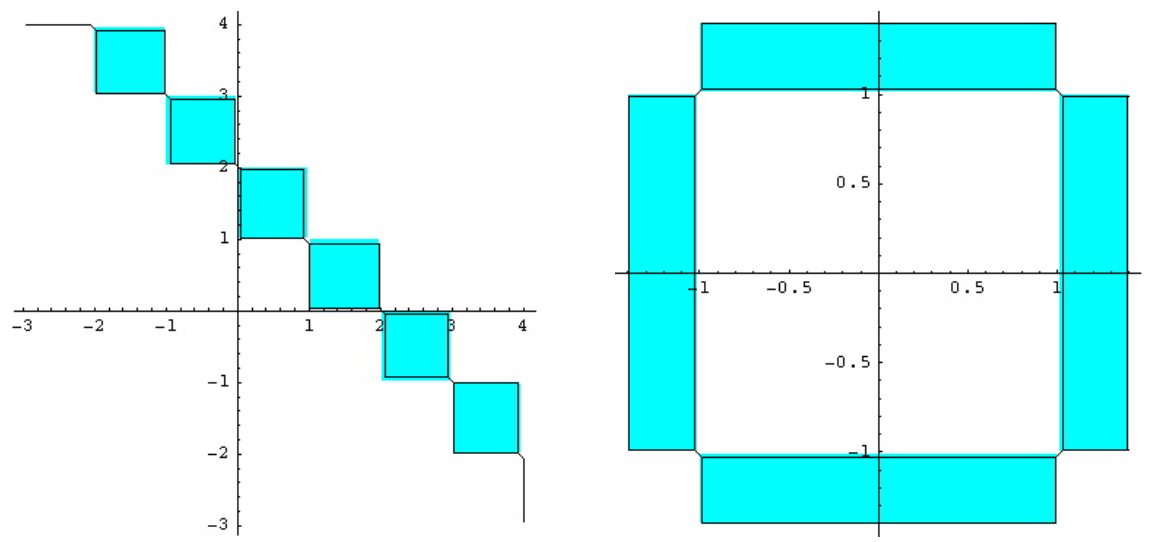

Fig. 2. Some examples of inequality solutions: (left) floor $(x)+f l o o r(y)=1$ on the square $[-4,4] \times[-4,4]$; (right) floor $\left(x^{2}\right)+\operatorname{floor}\left(y^{2}\right)=1$ on the square $[-2,2] \times[-2,2]$

The previous command, InequationPlot, can be generalized to inequalities involving complex numbers. The new command

ComplexInequationPlot [ineqs, $\{z,\{$ Rezmin, Rezmax $\},\{\operatorname{Imzmin}, \operatorname{Imz}$ max $\}\}$, opts]

displays the solution sets of the inequalities ineqs of complex numbers inside the square in the complex plane given by [Rezmin, Rezmax $] \times[$ Imzmin, Imzmax $]$. 
In this case, the functions appearing within the inequalities need to be realvalued functions of a complex argument, e.g. Abs, Re and Im. For example:

In $[6]:=$ ComplexInequationPlot $[\#,\{z,\{-2,3\},\{-3,3\}\}$, AspectRatio->
Automatic $] \& / @\left\{1<\operatorname{Abs}\left[z^{\wedge} 2-z^{+} 1\right]<4,1<\operatorname{Abs}\left[z^{\wedge} 2-2 z\right] / \operatorname{Abs}\left[z^{\wedge} 2+3\right]<4\right\}$

Out $[6]:=$ See Figure 3
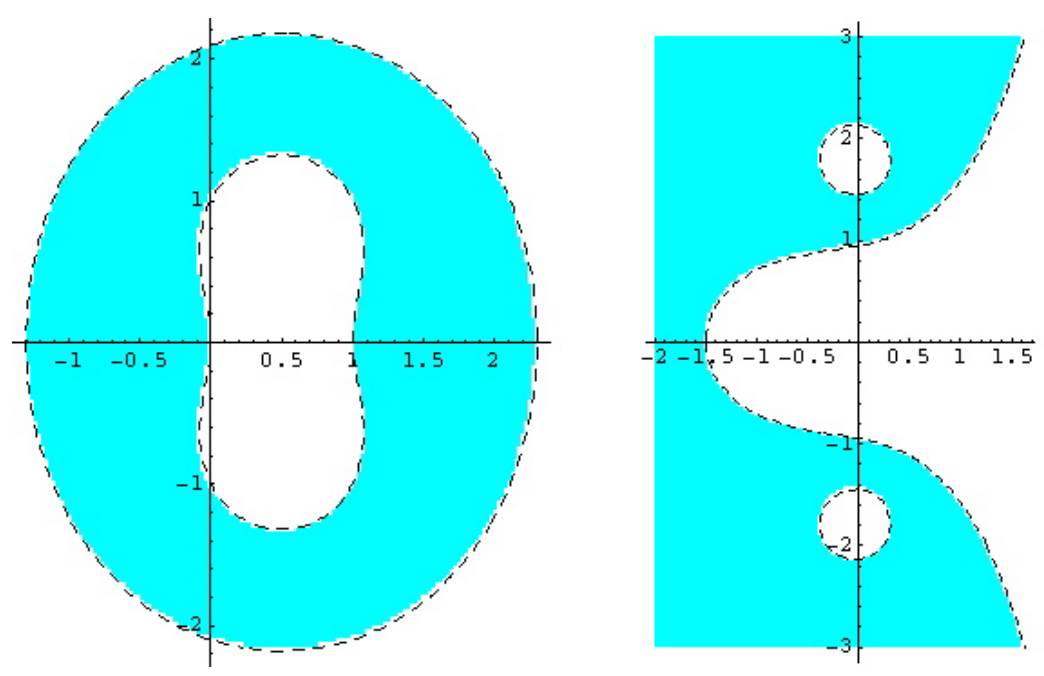

Fig. 3. Some examples of inequality solutions for $z \in \mathbb{C}$ such that $\operatorname{Re}(z) \in[-2,3]$ and $\operatorname{Im}(z) \in[-3,3]:$ (left) $1<\left\|z^{2}-z+1\right\|<4 ;$ (right) $1<\frac{\left\|z^{2}-2 z\right\|}{\left\|z^{2}+3\right\|}<4$

We would like to point out that the output obtained from the package is consistent with Mathematica's notation and results and the package provides a similar output for those cases already solved by Mathematica. Figure 4 illustrates this fact: on the left, the solutions provided by the standard Mathematica command InequalityPlot to the inequalities $x^{2}+y^{2} \leq 8 \wedge y^{2} \leq 2 x$ (top) and $x^{2}-y^{2} \geq 1 \bigwedge\left(x^{2}-1\right)^{3} y^{2} \leq 1$ (bottom) on $[0,3] \times[-3,3]$ and $[-3,3] \times[-2,2]$ respectively are displayed. On the right, the solutions obtained by using the new command InequationPlot are shown. As the reader can see, the new solution sets match perfectly those obtained from the standard Mathematica commands. 

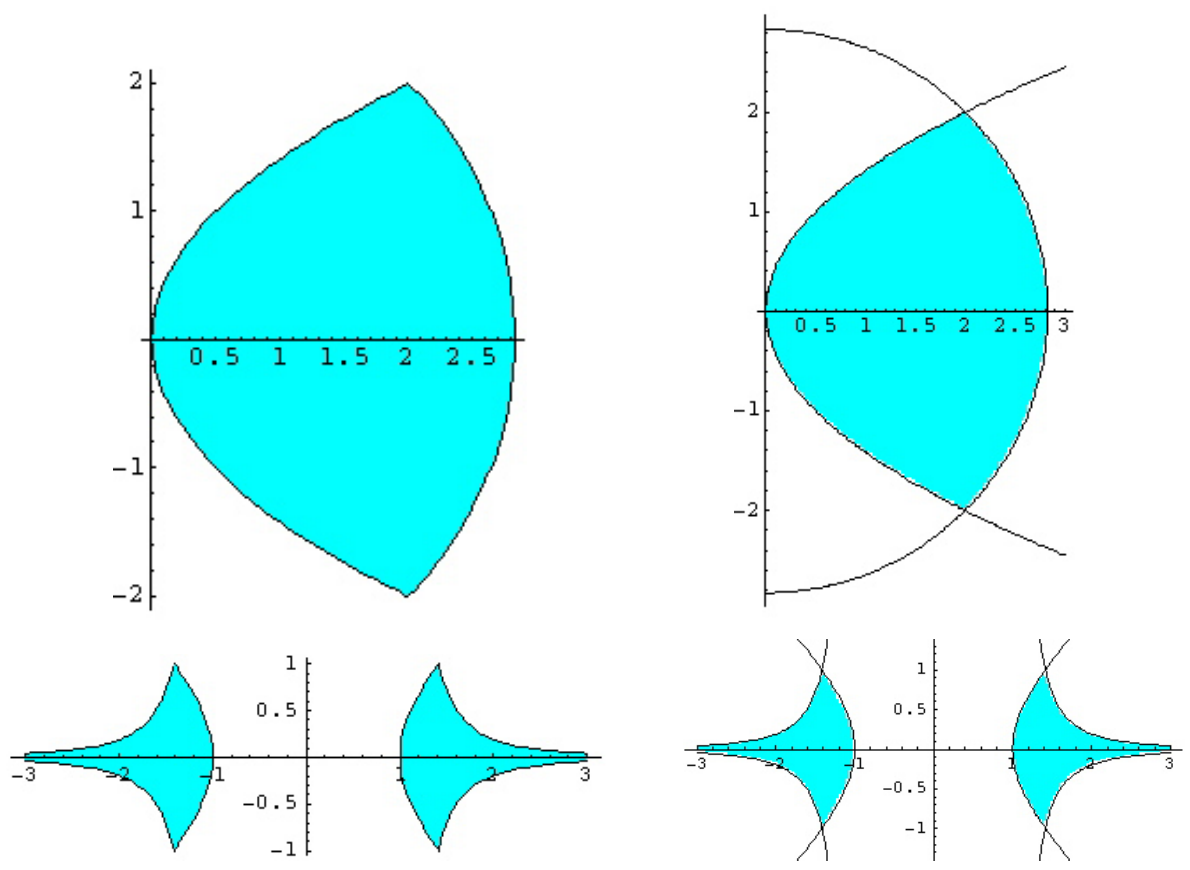

Fig. 4. Solution sets for the inequality systems: (top) $x^{2}+y^{2} \leq 8 \bigwedge y^{2} \leq 2 x$; (bottom) $x^{2}-y^{2} \geq 1 \bigwedge\left(x^{2}-1\right)^{3} y^{2} \leq 1$. The solutions have been obtained by applying: (left) the standard Mathematica command InequalityPlot; (right) the new command InequationPlot.
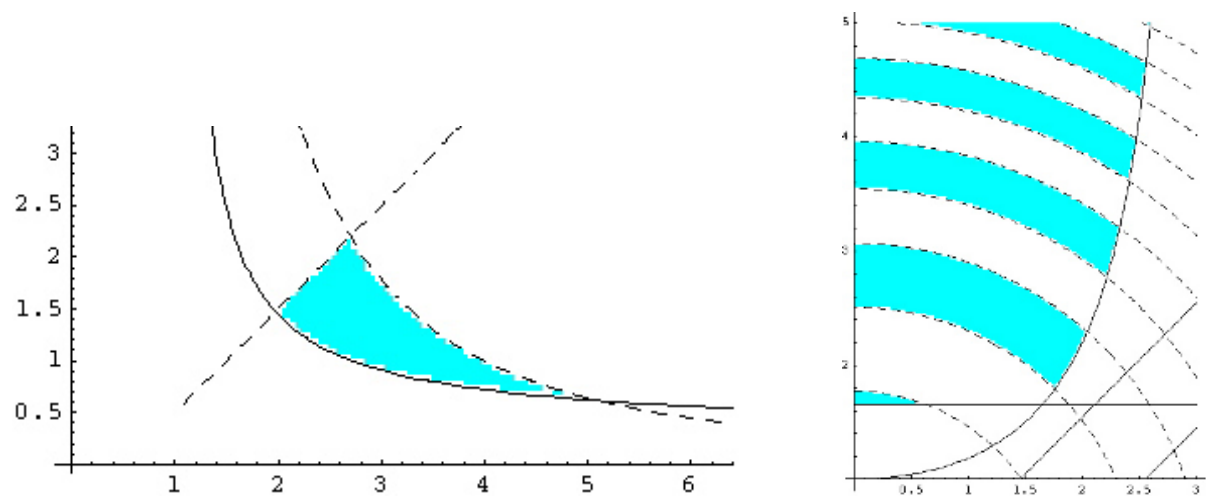

Fig. 5. (left, right) Solution sets for the inequality systems given by Eqns. (5) and (6) respectively 
The last example aims to show how complicated the inequality systems can be: in addition to include exponential, logarithmic and trigonometric functions, combinations and even compositions of these (and other) functions can also be considered. In Figure [5 the solutions sets of the inequality systems:

$$
\begin{gathered}
e^{y} \geq 1 \bigwedge \log (x) y \geq 1 \bigwedge x \sqrt{y}<4 \bigwedge x-y>\frac{1}{2} \\
\log (y) \geq \frac{1}{2} \bigwedge \sin (x) y \geq x \bigwedge \cos \left(e^{x-y}\right) \geq 0 \bigwedge \sin \left(x^{2}+y^{2}\right)>0 \\
\text { on }[1,10] \times[0,10] \text { and }[0,3] \times[1,5] \text { respectively are displayed. }
\end{gathered}
$$

\section{Conclusions and Further Remarks}

In this paper a new Mathematica package, InequationPlot, to solve real and complex inequalities and display their associated two-dimensional solution sets is introduced. The package extends Mathematica's capabilities by providing graphical solutions to many inequalities (such as those involving trigonometric, exponential and logarithmic functions) that cannot be solved by using the standard Mathematica commands and packages. The performance of the package has been illustrated by means of several interesting examples. In all the cases, the output obtained is consistent with Mathematica's notation and results and the package provides a similar output for those cases already solved by Mathematica. Perhaps the weakest feature of the package is the computation time, which is substantially higher than that usually required by Mathematica for solving the common cases. Further work will be developed in order to decrease this computation time and also to extend our results to the three-dimensional case. The obtained results will be reported elsewhere.

\section{References}

1. Beckenbach, E.F., Bellman, R.E.: An Introduction to Inequalities. Random House, New York (1961)

2. Brown, C.W.: Simple cylindrical algebraic decomposition construction and its applications. Journal of Symbolic Computation, 31 (2001) 521-547

3. Caviness, B.F., Johnson, J.R.: Quantifier Elimination and Cylindrical Algebraic Decomposition. Springer-Verlag, New York (1998)

4. Hardy, G.H., Littlewood, J.E., Pólya, G.: Inequalities (Second Edition). Cambridge University Press, Cambridge (1952)

5. Kazarinoff, N.D.: Geometric Inequalities. Random House, New York (1961)

6. McCallum, S.: Solving polynomial strict inequalities using cylindrical algebraic decomposition. The Computer Journal, 36(5) (1993) 432-438

7. Mitrinovic, D.S.: Analytic Inequalities. Springer-Verlag, New York (1970)

8. Mitrinovic, D.S., Pecaric, J.E., Volenec, V.: Recent Advances in Geometric Inequalities. Kluwer, Dordrecht (1989)

9. Strzebonski, A.: An algorithm for systems of strong polynomial inequalities. The Mathematica Journal, 4(4) (1994) 74-77

10. Strzebonski, A.: Solving algebraic inequalities. The Mathematica Journal, 7 (2000) 525-541 